\title{
Adaptive Homing and Distinguishing Experiments for Nondeterministic Finite State Machines
}

\author{
Natalia Kushik $^{1,2}$, Khaled El-Fakih ${ }^{3}$, and Nina Yevtushenko ${ }^{1}$ \\ ${ }^{1}$ Tomsk State University, Tomsk, Russia \\ ngkushik@gmail.com, ninayevtushenko@yahoo.com \\ ${ }^{2}$ Telecom SudParis, Evry, France \\ ${ }^{3}$ American University of Sharjah, Sharjah, UAE \\ kelfakih@aus.edu
}

\begin{abstract}
Adaptive experiments are well defined in the context of finite state machine (FSM) based analysis, in particular, in FSM based testing where homing and distinguishing experiments with FSMs are used in test derivation. In this paper, we define and propose algorithms for deriving adaptive homing and distinguishing experiments for non-initialized nondeterministic finite state machines (NFSM). For NFSMs, the construction of adaptive experiments is rather complex as the partition over produced outputs does not define a partition over the set of states but rather a collection of intersecting subsets, and thus, the refinement of such subsets is more difficult than the refinement of a partition. Given a complete non-initialized observable NFSM, we establish necessary and sufficient conditions for having adaptive homing and distinguishing experiments and evaluate the upper bound on the height of these experiments. Simple application examples demonstrating a proposed approach are provided.
\end{abstract}

Keywords: Nondeterministic finite state machine, conformance testing, adaptive homing and distinguishing experiments.

\section{Introduction}

Many methods are known for the development of experiments and conformance tests based on the specification given in the form of a finite state machine (FSM) [see, for example, 1-7]. In FSM-based testing, given a machine or an implementation under test (IUT) about which we lack some information, experiments are performed with the machine to deduce some lacked information. An experiment consists of applying input sequences to the machine, observing corresponding output responses and drawing the conclusion about the machine under test. An experiment is preset if input sequences are known before starting the experiment and an experiment is adaptive if at each step of the experiment the next input is selected based on previously observed outputs [3][8]. Well-known types of experiments include distinguishing and homing experiments which are used when deriving FSM based tests with the guaranteed fault coverage and those experiments are elaborated for different types of FSMs. An FSM is initialized if it has one initial state, otherwise, weakly-initialized or non-initialized. 
An FSM is observable if at each state the machine has at most one transition under a given input/output pair. Given an FSM, assuming that the initial state is unknown, a distinguishing experiment determines the initial state of the FSM, i.e., a state of the FSM before the experiment and such an experiment is widely used when checking the correspondence between transitions of an IUT and those of the specification FSM. A homing experiment identifies the final state reached at the end of the experiment and it is used when deriving a checking sequence for non-initialized FSMs $[9,10]$.

Ongoing research on preset and adaptive homing experiments for deterministic FSMs started since the seminal paper on "gedanken experiments" by Moore [8]. For information and surveys on FSM-based experiments and some related algorithms, a reader may refer to $[4,5,11]$; in particular, in $[3,5]$ a reader can find methods for deriving preset and adaptive distinguishing experiments for deterministic FSMs with corresponding evaluation of the length of these experiments. Preset homing experiments are considered in $[3,4,12,13]$. Derivation of minimal length preset homing sequences can be done using the homing tree method introduced by Gill [3] and reported in details in Kohavi [4]. Any deterministic complete reduced FSM with $n$ states has been shown to have a homing sequence of length up to $n(n-1) / 2$ and Hibbard [14] showed that deterministic machines require adaptive homing sequences with length of the same order. There is also some work devoted to the derivation of a synchronizing sequence that takes the FSM from any initial state to the same state independent of produced output sequences. As in this case, outputs are not important, most researchers derive such sequences for corresponding automata where only input actions are considered. A related detailed survey is given by Sandberg in [15]. Parallel algorithms for related problems are surveyed by Ravikumar [see, for example, 16].

In this paper, we consider homing and distinguishing experiments with nondeterministic FSMs, as nowadays, analysis and testing of nondeterministic systems are capturing a lot of attention. Preset distinguishing and homing experiments for nondeterministic FSMs are considered in [17, 18]. In particular, Spitsyna et al. [17] presented the method for deriving a sequence that separates two initialized nondeterministic FSMs. An input sequence is a separating sequence of two FSMs if the sets of output sequences produced by the NFSMs to the input sequence do not intersect [19]. Kushik et al. [18] showed that differently from deterministic FSMs a homing sequence does not necessarily exist for a complete reduced nondeterministic FSM and proposed an algorithm for deriving a preset homing sequence for a given nondeterministic FSM when such a sequence exists. A tight lower bound on a shortest preset homing sequence is shown to be of the order $2^{n^{2}}$ where $n$ is the number of states of the nondeterministic FSM. Moreover, it has been shown [20] that there exists a special class of FSMs with $n$ states and $(n-1)$ inputs, for which a shortest homing sequence has the length $2^{n-1}$ - 1. i.e., its length is exponential not only with respect to the number of FSM states but also the number of FSM transitions. Zhang and Cheung studied related problems when deriving transfer and distinguishing trees for observable nondeterministic FSMs with probabilistic and weighted transitions [21].

Adaptive experiments with nondeterministic FSMs are considered in [22-25]. In [23], Petrenko and Yevtushenko introduced the notion of a test case for describing an adaptive experiment as an initialized observable FSM with an acyclic transition diagram 
such that at each non-deadlock state only one input is defined with all possible outputs. A representation of a test case using the same formal model is widely used for transition systems such as LTS, input/output automata etc. [see, for example 26]. Such definition of a test case allows defining distinguishing/checking/homing test cases based on the properties of the intersection of a transition system under experiment and a given test case. In [22-25] it is shown how a distinguishing test case can be derived for two states of NFSMs when such a distinguishing test case exists. In particular, Alur et al. [22] show that the length of a shortest adaptive distinguishing test case that distinguishes two states of an observable nondeterministic FSMs with $n$ states is at most $n(n-1) / 2$. Petrenko and Yevtushenko [25] consider a set of adaptive test cases which have three parts: a preamble for reaching an appropriate state, a traversal input/output sequence and a state identifier. In this case, the length of an identifier can be optimized when distinguishing not two but several states with the same distinguishing test case. In addition, a checking sequence derived for a non-initialized FSM $[9,10]$ also can be adaptive but in this case, an adaptive homing experiment should be performed before applying such a sequence. Gromov et al. [27] and El-Fakih et al. [28] presented adaptive experiments for two timed nondeterministic observable FSMs.

In this paper, we consider adaptive homing and distinguishing experiments for non-initialized nondeterministic finite state machines. Similar to many other papers described above, an adaptive experiment is represented by a test case that is an initialized observable FSM with the acyclic flow diagram where only one input is defined at each intermediate state. Lee and Yannakakis [5] proposed an approach for deriving an adaptive distinguishing sequence of a deterministic FSM that is based on refining a partition of the set of states based on different outputs. In this paper, we deal with nondeterministic FSMs and unlike [5], the output partition defines not a partition of the set of states but rather a set system, which is a collection of intersecting subsets, for which it is difficult to define a corresponding refinement. For this reason, in this paper, necessary and sufficient conditions for having adaptive homing/distinguishing test cases are established based on extending the notion of $k$-r-distinguishability of two states [29] to subsets of states and an algorithm for deriving a homing/distinguishing adaptive test case with minimal length is proposed. The upper bound on the length of homing/distinguishing experiments is determined and an example illustrating that the upper bound seems to be tight for the length of distinguishing test cases is presented.

This paper is organized as follows. Section 2 includes preliminaries. Homing and distinguishing test cases with related properties are introduced in Section 3. Section 4 contains an approach for deriving a homing/distinguishing test case with corresponding statements about the complexity. Section 5 concludes the paper.

\section{Preliminaries}

In this paper, we consider experiments with weakly initialized Finite State Machines. A weakly initialized Finite State Machine (FSM) S is a 5-tuple $\left(S, I, O, h_{\mathrm{S}}, S^{\prime}\right)$, where $S$ is a finite set of states with the set $S^{\prime} \subseteq S$ of initial states; $I$ and $O$ are finite nonempty disjoint sets of inputs and outputs, respectively; $h_{\mathrm{S}} \subseteq S \times I \times O \times S$ is a 
transition relation, where a 4-tuple $\left(s, i, o, s^{\prime}\right) \in h_{\mathrm{S}}$ is a transition. If $\left|S^{\prime}\right|=1$ then the FSM $S$ is an initialized FSM. An input $i \in I$ is a defined input at state $s$ of $S$ if there exists a transition $\left(s, i, o, s^{\prime}\right) \in h_{\mathrm{S}}$ for some $s^{\prime} \in S$ and $o \in O$.

An FSM S $=\left(S, I, O, h_{\mathrm{S}}, S^{\prime}\right)$ is complete if for each pair $(s, i) \in S \times I$ there exists $\left(o, s^{\prime}\right) \in O \times S$ such that $\left(s, i, o, s^{\prime}\right) \in h_{\mathrm{S}}$. FSM S is nondeterministic if for some pair $(s, i) \in S \times I$, there exist at least two transitions $\left(s, i, o_{1}, s_{1}\right),\left(s, i, o_{2}, s_{2}\right) \in h_{\mathrm{S}}$, such that $o_{1} \neq o_{2}$ or $s_{1} \neq s_{2}$. FSM $S$ is observable if for each two transitions $\left(s, i, o, s_{1}\right)$, $\left(s, i, o, s_{2}\right) \in h_{\mathrm{S}}$ it holds that $s_{1}=s_{2}$. FSM S is single-input if at each state there is at most one defined input at the state, i.e., for each two transitions $\left(s, i_{1}, o_{1}, s_{1}\right),\left(s, i_{2}, o_{2}\right.$, $\left.s_{2}\right) \in h_{\mathrm{S}}$ it holds that $i_{1}=i_{2}$, and FSM S is output-complete if for each pair $(s, i) \in$ $S \times I$ such that the input $i$ is defined at state $s$, there exists a transition from $s$ with $i$ for every output in $O[21,25]$.

A trace of $\mathrm{S}$ at state $s$ is a sequence of input/output pairs of sequential transitions starting from state $s$. Let $\operatorname{Tr}(\mathrm{S} / s)$ denote the set of all traces of $\mathrm{S}$ at state $s$ including the empty trace and let $\operatorname{Tr}\left(\mathrm{S} / S^{\prime}\right)$ denote the union of $\operatorname{Tr}(\mathrm{S} / s)$ over all states $s \in S^{\prime}$. As usual, for state $s$ and a sequence $\gamma \in(I O)^{*}$ of input-output pairs, next_states $(s, \gamma)$ denotes the set of all states that are reached from $s$ by $\gamma$. If $\gamma$ is not a trace at state $s$ then the set next_states $(s, \gamma)$ is empty; otherwise, each state of the set next_states $(s, \gamma)$ is a $\gamma$-successor of state $s$. For an observable FSM S, $\mid$ next_states $(s, \gamma) \mid \leq 1$ for any string $\gamma$ $\in(I O)^{*}$. Given a nonempty subset $S^{\prime}$ of states of the FSM S and $\gamma \in(I O)^{*}$, the set next_states $\left(S^{\prime}, \gamma\right)$ is the union of the sets next_states $(s, \gamma)$ over all $s \in S^{\prime}$ and this set is a $\gamma$-successor of the set $S^{\prime}$. An FSM S is acyclic if the set $\operatorname{Tr}\left(\mathrm{S} / S^{\prime}\right)$ is finite, i.e., the FSM transition diagram has no cycles.

To characterize the common behavior of two weakly initialized machines, we extend the operation of the intersection of initialized FSMs as follows. Given two complete FSMs $\mathrm{S}$ and $\mathrm{P}$ with the sets $S^{\prime}$ and $P^{\prime}$ of initial states, the intersection $\mathrm{S} \cap \mathrm{P}$ is the connected FSM Q such that states of $\mathrm{Q}$ are pairs $(b, c)$ of sets of states of FSMs $\mathrm{S}$ and $\mathrm{P}$, the initial state of $\mathrm{Q}$ is $\left(S^{\prime}, P^{\prime}\right)$, and $h_{\mathrm{Q}}$ is the smallest set derived using the following rule: Given state $(b, c), b \subseteq S$ and $c \subseteq P$, and an input/output pair $i / o$, the FSM Q has a transition $\left((b, c), i, o,\left(b^{\prime}, c^{\prime}\right)\right)$ if there exist states $s \in b$ and $p \in c$ with an outgoing transition labeled by the pair $i / o$, and $b^{\prime}$ and $c^{\prime}$ are $i / o-$ successors of subsets $b$ and $c$. By definition, the FSM $\mathrm{S} \cap \mathrm{P}$ is observable even for non-observable FSMs $\mathrm{S}$ and $\mathrm{P}$.

As an example of the FSM intersection, consider FSMs P (Fig. 1) and S (Fig. 2). FSM $P$ is an initialized FSM while $S$ has three initial states marked in bold. The intersection $\mathrm{S} \cap \mathrm{P}$ is shown in Fig. 3. As usual, the intersection of two weakly initialized FSMs describes the common behavior of component FSMs, and in addition, it also provides some information about the structure of their transition sets. For example, a state of the intersection provides information about which states of the corresponding machines are reachable from the initial states under a corresponding trace. In fact, the following proposition holds.

Proposition 1. Given FSMs S and P with the sets $S^{\prime}$ and $P^{\prime}$ of initial states and state $(b, c)$ of the intersection $\mathrm{S} \cap \mathrm{P}$ that is reachable from the initial state under a trace $\gamma$, the set $b$ is the $\gamma$-successor of the set $S^{\prime}$ while the set $c$ is the $\gamma$-successor of the set $P^{\prime}$. 
As in this paper we consider adaptive experiments with nondeterministic FSMs, in order to identify a state of a weakly initialized FSM before or after the experiment, a finite input sequence is applied to an FSM under experiment where the next input (except of the first one) of the sequence is determined based on the output of the FSM produced to the previous input. Formally, such an experiment can be described using a single-input output-complete FSM with an acyclic transition graph and similar to $[23,25]$ we refer to such an FSM as a test case.

Given an input alphabet $I$ and an output alphabet $O$, a test case $T C(I, O)$ is an initially connected single-input output-complete observable initialized FSM $\mathrm{P}=\left(P, I, O, h_{\mathrm{P}}, p_{0}\right)$ with acyclic transition graph. By definition, if $|I|>1$ then a test case is a partial FSM.

A test case $\mathrm{P}$ over alphabets $I=\{a, b\}$ and $O=\{0,1\}$ is shown in Fig. 1.

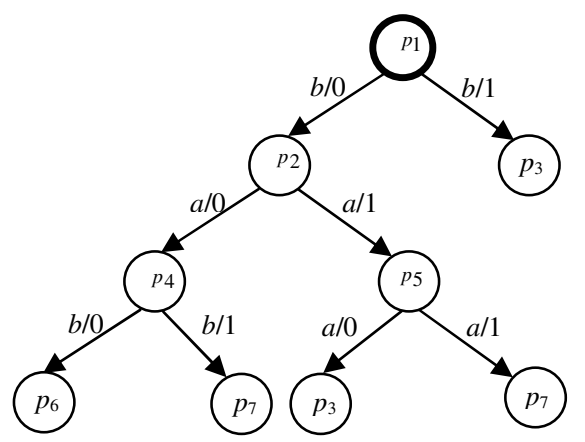

Fig. 1. A test case $\mathrm{P}$ over alphabets $I=\{a, b\}$ and $O=\{0,1\}$

A test case $T C(I, O)$ over alphabets $I$ and $O$ defines an adaptive experiment with any FSM S over the same alphabets. As an example, consider the test case $\mathrm{P}$ in Fig. 1. An adaptive experiment with an FSM S over alphabets $I=\{a, b\}$ and $O=\{0$, $1\}$ is conducted using $\mathrm{P}$ as follows. At the first step the input $b$ is applied to $\mathrm{S}$ as this input is the only input defined at the initial state of P. If the output of the FSM $S$ to this input is 1 , then the experiment is over, since we reach the deadlock state $p_{3}$ of $\mathrm{P}$. If the FSM $S$ produces the output 0 to input $b$ then the experiment is not over, since the test case $\mathrm{P}$ enters the intermediate state $p_{2}$ where the single input $a$ is defined. As this input does not take the test case to a deadlock state, the next input which is also $a$ is applied. If the output to $a$ is 0 then the next input is $b$; otherwise, the next input is $a$. For this example, the length of a longest trace of the test case is three, i.e., at most three inputs are applied during this adaptive experiment.

In general, given a test case $P$, the length of the test case $P$ is determined as the length of the longest trace from the initial state to a deadlock state of $\mathrm{P}$ and it specifies the length of the longest input sequence that can be applied to an FSM S during the experiment that is also often called the height of the adaptive experiment. As usual, for testing, one is interested in deriving a test case (experiment) with minimal length (height). 


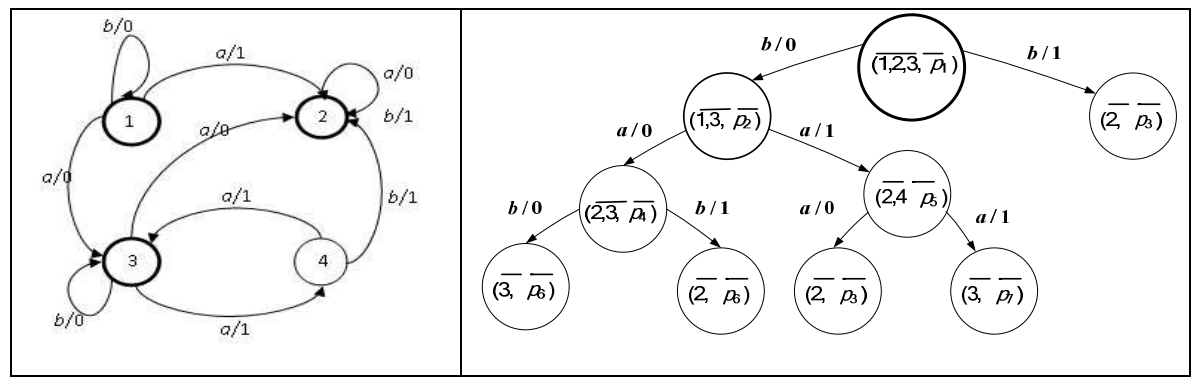

Fig. 2. FSM S with three initial states

Fig. 3. The intersection $\mathbf{S} \cap \mathbf{P}$

\section{$3 \quad$ Homing and Distinguishing Test Cases}

In this section, we define the notions of homing and distinguishing test cases that can be used in the context of adaptive testing of non-initialized nondeterministic observable FSMs. A homing (distinguishing) experiment allows determining the unknown current (initial) state of the machine under experiment. Hereafter, if not stated otherwise, we consider an FSM under experiment to be a weakly initialized complete nondeterministic observable FSM and in the following sections we propose a method for deriving homing and distinguishing test cases for an FSM and we also determine the upper bound on minimal length of a test case.

Given a complete observable FSM S over input and output alphabets $I$ and $O$, let $S^{\prime}$ be the set of initial states of FSM S. A test case P is a homing test case for FSM S if for each deadlock state $(b, c)$ of the intersection $\mathrm{S} \cap \mathrm{P}$, the set $b$ is a singleton.

A test case $\mathrm{P}$ is a distinguishing test case for $\mathrm{FSM} \mathrm{S}=\left(S, I, O, h_{\mathrm{S}}, S^{\prime}\right)$ if (1) for each deadlock state $(b, c)$ of the intersection $\mathrm{S} \cap \mathrm{P}$ subset $b$ is a singleton, and (2) for each transition $\left((b, c), i, o,\left(b^{\prime}, c^{\prime}\right)\right)$ of the intersection $\mathrm{S} \cap \mathrm{P}$ the subset $b$ does not have two different states which have the same $i / o-$ successor, i.e.,

$\forall s_{1}, s_{2} \in b\left(\left(s_{1}, i, o, s^{\prime}\right) \in h_{\mathrm{S}} \&\left(s_{2}, i, o, s^{\prime}\right) \in h_{\mathrm{S}} \Rightarrow s_{1}=s_{2}\right)$.

If there exists a homing (distinguishing) test case for the FSM then the set is $S^{\prime}$ is a homing (distinguishing) set and the test case $\mathrm{P}$ is a homing (distinguishing) test case for the set $S^{\prime}$ or the test case P homes (distinguishes) states of the set $S^{\prime}$.

According to the above definitions, the following statement holds.

Proposition 2. Given a weakly initialized observable FSM S, each distinguishing test case for $\mathrm{S}$ is also a homing test case. However, the converse is not necessarily true.

Example. As an example of homing and distinguishing test cases, consider a weakly initialized FSM S in Fig. 2 and the test case P in Fig. 1. By direct inspection, one can assure that each deadlock state of the intersection $\mathrm{S} \cap \mathrm{P}$ (Fig. 3) is labeled by a pair of singletons and each two different states of any subset $b$ such that $(b, c)$ labels an intermediate state of the intersection do not have the same $i / o$-successor. Thus, the set $\{1,2,3\}$ is not only a homing but also a distinguishing set and the test case in Fig. 1 is a homing test case and a distinguishing test case for FSM S. For example, if the output 1 
is produced to the input $b$ at the initial state of the FSM S then the FSM reaches state 2 after experiment and we certainly know that the initial state before the experiment was 2. However, we later show that the FSM S has a shorter homing test case.

A homing (distinguishing) test case is used for representing a homing (distinguishing) adaptive experiment with a nondeterministic FSM. An adaptive homing (distinguishing) experiment has two steps. At the first step a finite input sequence is applied to an FSM under experiment where the next input (except of the first one) significantly depends on the output of the FSM produced to the previous input. At the next step, after observing a produced output sequence, the conclusion is drawn about a state of the FSM after (before) the experiment and such a state has to be unique for the homing (distinguishing) experiment. If all the traces of a test case have the same input projection, then the test case defines a preset input sequence and a corresponding adaptive experiment becomes a preset experiment. In the same way, when having a homing or separating sequence for a given FSM a corresponding test case can be derived by augmenting this sequence with all possible output sequences.

Propositions 3 and $3^{\prime}$ state necessary and sufficient conditions for a test case to be a homing and/or a distinguishing test case. As the proofs are almost the same, we only prove Proposition 3.

Proposition 3. Given a weakly initialized observable FSM S $=\left(S, I, O, h_{\mathrm{S}}, S^{\prime}\right)$, a test case $\mathbf{P}$ is a homing test case for $\mathrm{S}$ if and only if every trace of $\mathrm{P}$ from the initial state to a deadlock state that is a trace at two different initial states of the set $S^{\prime}$ takes the FSM S from these initial states to the same state.

Proof. $\Rightarrow$ Let $\mathrm{P}$ be a homing test case for FSM S $=\left(S, I, O, h_{\mathrm{S}}, S^{\prime}\right)$. Suppose that $\exists s_{1}$, $s_{2} \in S^{\prime} \exists \alpha \in I^{*} \exists \beta \in O^{*}\left(\beta \in \operatorname{out}\left(s_{1}, \alpha\right) \cap \operatorname{out}\left(s_{2}, \alpha\right)\right)$ and the trace $\alpha / \beta$ takes the FSM $\mathrm{P}$ from the initial to a deadlock state $(b, c)$. The deadlock state $(b, c)$ of the intersection $\mathrm{S} \cap \mathrm{P}$ contains $\alpha / \beta$-successors of states $s_{1}, s_{2}$ (Proposition 1 ), i.e., $\alpha / \beta$ successors of these states coincide.

$\Leftarrow$ Consider a trace $\alpha / \beta$ that takes the FSM P from the initial state to a deadlock state $p$. Let $s_{1}, s_{2} \in S^{\prime}$ be initial states where the trace $\alpha / \beta$ can be executed and state $s^{\prime}$ is an $\alpha / \beta$-successor of states $s_{1}$ and $s_{2}$ (since FSM $S$ is observable). By the intersection construction, the FSM $\mathrm{S} \cap \mathrm{P}$ has a deadlock state $\left(\overline{s^{\prime}}, \bar{p}\right)$ where both items are singletons.

Proposition 3'. Given a weakly initialized observable FSM $S=\left(S, I, O, h_{\mathrm{S}}, S^{\prime}\right)$, a test case $P$ is a distinguishing test case for FSM $S$ if and only if every trace from the initial state to a deadlock state of $\mathrm{P}$ is a trace at only one initial state of the set $S^{\prime}$.

According to the above Propositions 3 and $3^{\prime}$, given a weakly initialized FSM S there exists an adaptive homing (distinguishing) experiment for the FSM S if and only if the FSM S has a homing (distinguishing) test case.

Here we note that the above propositions establish one-to-one correspondence between homing/distinguishing test cases and adaptive homing/distinguishing experiments only for observable FSMs; for non-observable FSMs, the definition of a distinguishing test case that corresponds to an adaptive distinguishing experiment should be modified. If a given FSM S is observable then according to the following 
proposition there is a simple way to check whether there exists a corresponding homing experiment.

Proposition 4. Given a weakly initialized observable FSM S with the set $S^{\prime}$ of initial states, there exists a homing test case for the set $S^{\prime}$ of $S$ if each subset $\left\{s_{i}, s_{j}\right\}$ of two states of $\mathrm{S}$ is a homing set.

Proof. We prove this proposition by construction. Let for each two different states $s_{i}$ and $s_{j}, s_{i} \neq s_{j}$, of $\mathrm{S}$ there exist a homing test case $\mathrm{P}_{i, j}$. Without loss of generality we assume that $S^{\prime}=\left\{s_{1}, . ., s_{m}\right\}$. Consider a homing test case $\mathrm{P}_{1,2}$ for the set $\left\{s_{1}, s_{2}\right\}$ and derive a test case $\mathrm{P}_{1,2,3}$ by adding the state $s_{3}$ into the set labeling the initial state of $\mathrm{P}_{1,2}$ and obtain $\mathrm{P}_{1,2,3}$ that includes all the transitions of $\mathrm{P}_{1,2}$. Subsets of states that label deadlock states of $S \cap P_{1,2,3}$ are not necessarily singletons but they contain at most two states, since FSM is observable. Each pair of different states of $\mathrm{S}$ is homing, thus, for each deadlock state $\left(\overline{s_{i}, s_{j}}, \bar{p}\right)$ of $\mathrm{S} \cap \mathrm{P}_{1,2,3}$ we concatenate the initial test case $\mathrm{P}_{1,2,3}$ with the corresponding test case $\mathbf{P}_{i, j}$. Proceeding in the same way we derive test case $\mathbf{P}_{1,2, \ldots, m}$. By construction, the derived test case is homing for the FSM S.

Corollary. Given a weakly initialized observable FSM S with the set $S$ of initial states, there exists a homing test case for FSM $S$ if and only if there exists a homing test case for each two different states $s_{i}$ and $s_{j}$ of $S$.

Proposition 4'. Given a nondeterministic observable $\mathrm{FSM} \mathrm{S}=\left(S, I, O, h_{\mathrm{S}}, S\right)$, if for each two different states $s_{i}$ and $s_{j}$ of $S$ there exists a homing test case, then there exists a homing test case for FSM S with length $O\left(n^{3}\right)$.

Proof. Each pair of states of an observable FSM can be homed by a sequence of inputs of length at most $C_{n}{ }^{2}$ that covers in the worst case all pairs of different FSM states. The number of states to be appended at each step of the procedure used in the proof of Proposition 4 equals $(n-1)$. Thus, the maximal length of the trace in the test case $\mathrm{P}_{1,2, \ldots, n}$ has length at most of the order $n^{3}$.

In other words, if each pair of states of an observable FSM S with $n$ states can be adaptively homed then there exists a homing test case for the set $S$ of FSM states and the length of this test case is of the order $n^{3}$. The proof of Proposition 4 proposes a procedure for deriving such a homing test case when the conditions of the proposition hold.

Example. As an example of applying the construction stated in Proposition 4, consider the FSM $S$ with the set of initial states $S^{\prime}=\{1,2,3\}$ in Fig. 4 below. FSM $S$ is complete and observable. We derive a homing test case for this FSM by deriving a test case $\mathrm{P}_{i, j}$ for each pair $(i, j), i \neq j$, of the FSM states and then derive a test case for the set $S^{\prime}$. The set of transitions of $\mathrm{P}_{12}$ equals $\left\{\left(\overline{1,2}, i_{1}, o_{1}, \overline{1}\right),\left(\overline{1,2}, i_{1}, o_{2}, \overline{2}\right)\right\}$; the set of transitions of $\mathrm{P}_{13}$ equals $\left\{\left(\overline{1,3}, i_{3}, o_{1}, \overline{1}\right),\left(\overline{1,3}, i_{3}, o_{2}, \overline{3}\right)\right\}$; and the set of $\mathrm{P}_{23}$ transitions equals $\left\{\left(\overline{2,3}, i_{2}, o_{1}, \overline{2}\right),\left(\overline{2,3}, i_{2}, o_{2}, \overline{3}\right)\right\}$. We add state 3 to the pair $\overline{1,2}$, include transitions of $\mathrm{P}_{12}$ into $\mathrm{P}_{123}$ and concatenate the obtained test case with appropriate test cases for subsets of two states. More precisely, this is done as follows. First, we consider input $i_{1}$ at state 3 for which a single output $o_{1}$ can be obtained, thus, 
the corresponding transitions at state $\overline{1,2,3}$ of $\mathrm{P}_{123}$ are $\left\{\left(\overline{1,2,3}, i_{1}, o_{1}, \overline{1,2}\right),\left(\overline{1,2,3}, i_{1}\right.\right.$, $\left.\left.o_{2}, \overline{2}\right)\right\}$. At state $\overline{1,2}$, we append corresponding transitions from the test case $\mathrm{P}_{12}$ and obtain the set of transitions for $\mathrm{P}_{123}:\left\{\left(\overline{1,2,3}, i_{1}, o_{1}, \overline{1,2}\right),\left(\overline{1,2,3}, i_{1}, o_{2}, \overline{2}\right),\left(\overline{1,2}, i_{1}, o_{1}\right.\right.$, $\left.\overline{1}),\left(\overline{1,2}, i_{1}, o_{2}, \overline{2}\right)\right\}$. The length of such adaptive homing test case equals two. However, if we selected an input $i_{3}$ when deriving the set of transitions of $\mathrm{P}_{12}$ a corresponding adaptive homing test case for the set $S^{\prime}=\{1,2,3\}$ would have length 1 .

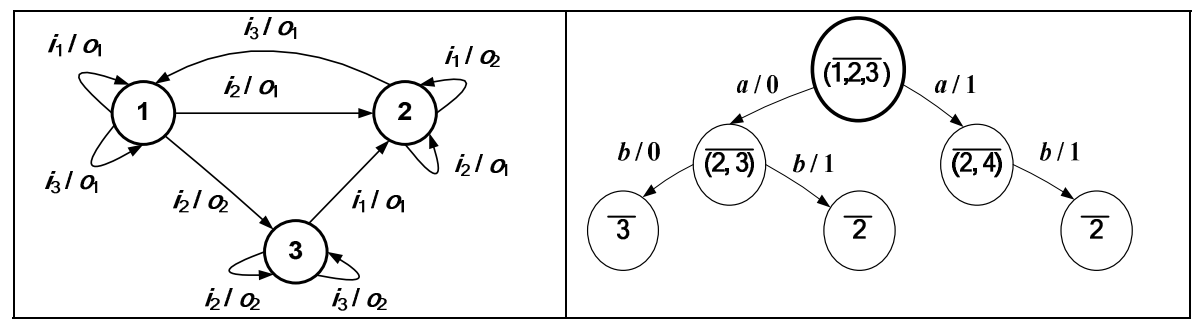

Fig. 4. FSM S

Fig. 5. Homing test case $P$ for the FSM in Fig. 2

Thus, we observe that when deriving an adaptive test case using the above procedure the length of a returned test case significantly depends on the enumeration of states of the set $S^{\prime}$ as well as on selected inputs at each step and therefore, does not guarantee the derivation of a homing test case of minimal length. Moreover, such a procedure does not guarantee the homing test derivation when there exists a pair of two different states of the FSM that is not homing. In the following we propose an algorithm that returns a homing test case with minimal length for a given FSM.

We also note that a proposition similar to Proposition 4 does not hold for a distinguishing test case. That is, given an observable FSM where each pair of two different states has a distinguishing test case, there is no guarantee that there exists a distinguishing test case for FSM S.

In order to derive a homing/distinguishing test case with minimal length, we introduce the notion of $k$-homing/k-distinguishing sets not only for pairs but for arbitrary subsets of states.

A subset $g$ of states of an observable FSM S is 0-homing if $g$ is a singleton. Let all $(k-1)$-homing sets, $k>0$, be already defined. The subset $g$ is a $k$-homing set if (1) $g$ is a $(k-1)$-homing, or (2) there exists an input $i \in I$, such that for each $o \in O$, the set of $i / o$-successors of states of $g$ is either empty or is a $(k-1)$-homing set.

In order to define a distinguishing test case for an observable FSM, the previous definition can be used, where the notion of $k$-homing is replaced by $k$-distinguishing and a subset $g$ of an FSM S $=\left(S, I, O, h_{\mathrm{S}}, S^{\prime}\right)$ is called a $k$-distinguishing set if (1) applies, i.e., $g$ is a $(k-1)$-distinguishing set, and for (2) as previously, there exists an input $i \in I$, such that for each $o \in O$, the set of $i / o$-successors of states of $g$ is either empty or is a $(k-1)$-distinguishing set, and in addition, the $i / o$-successors of two different states of $g$ do not coincide. 
Proposition 5. Given a weakly initialized observable complete FSM S with the set $S^{\prime}$ of the initial states, the set $S^{\prime}$ is $k$-homing/ $k$-distinguishing, $k>0$, if and only if there exists a homing/distinguishing adaptive experiment of height $k$ for the weakly initialized FSM $\mathrm{S}=\left(S, I, O, h_{S}, S^{\prime}\right)$. If $S^{\prime}$ is $k$-homing/ $k$-distinguishing, $k>0$, but is not $(k-1)$-homing/(k-1)-distinguishing then $k$ is the minimal height of a corresponding adaptive experiment.

Proof. We provide the proof of the Proposition for the case of a homing experiment as for the distinguishing test case the proof is almost the same.

$\Rightarrow$ We use the induction on $k$. Let $k=1$. By definition, there exists an input $i \in I$, such that for each $o \in O$, the set of $i / o$-successors of states of $S^{\prime}$ is either empty or is a 0 -homing set (a singleton), i.e., there exists an adaptive experiment of length 1 for the $\mathrm{S}=\left(S, I, O, h_{S}, S^{\prime}\right)$.

Let the statement hold for all $k \leq K$ and $S^{\prime}$ is not a $K$-homing set but $S^{\prime}$ is a $(K+1)$ homing set. In this case, by definition, there exists an input $i \in I$, such that for each $o \in O$, the set of $i / o$-successors of states of $S^{\prime}$ is either empty or is a $K$-homing and according to the induction assumption, each if $i / o$-successors can be homed by an adaptive experiment of length at most $K$. Thus, $S^{\prime}$ can be homed by an adaptive experiment of length at most $(K+1)$.

$\Leftarrow$ Suppose there exists a test case $\mathrm{P}$ of a height $k>1$. By definition, the states of $\mathrm{P}$ that have transitions to deadlock states are 1-homing. Thus, the states of $P$ that are connected to these states are 2-homing, etc. The states of $P$ that are connected to the deadlock states via a sequence of length $k$ are $k$-homing.

Based on Propositions 3, 3' and 5, the following proposition holds.

Proposition 6. Given a set $S^{\prime},\left|S^{\prime}\right|>1$, of states of an observable FSM S, the set $S^{\prime}$ is homing/distinguishing if and only if $S^{\prime}$ is $k$-homing/ $k$-distinguishing for some $k>0$.

\section{Deriving Homing and Distinguishing Test Cases}

Based on the notion of $k$-homing sets, Procedure 1 given below can be used for deriving a homing/distinguishing test case for a homing/distinguishing set $S^{\prime}$ of states of a given observable FSM S. If the set $S^{\prime}$ is not homing/distinguishing then the states of the set $S^{\prime}$ cannot be homed/distinguished by an adaptive experiment. The main idea of the procedure below is to iteratively derive subsets of states that are homed/distinguished by adaptive applying an input sequence of the length $j \in\{1,2$, $\ldots, k\}$. The set of corresponding subsets we hereafter denote as $Q_{j}$. If for some $j$ the $Q_{j}$ is empty and the subset $S^{\prime} \notin \bigcup_{k=1}^{j-1} Q_{j}$ then a fixed point is reached and a homing experiment for the FSM S does not exist. The states of a test case under construction are labeled by subsets of $\mathrm{S}$ states. We describe a procedure for a homing test case, since for a distinguishing test case the procedure is almost the same. 


\section{Procedure 1: Deriving a homing test case for a subset $S^{\prime}$ of states of an FSM S}

Input: Complete observable nondeterministic weakly initialized FSM $\mathrm{S}=\left(S, I, O, h_{S}, S^{\prime}\right),\left|S^{\prime}\right|>1$

Output: A homing test case P with minimal length for the subset $S^{\prime}$ of FSM S or a message "there is no adaptive homing experiment for the subset $S$ "

Step-1: $j:=0$

Derive the set $Q_{j}$ of all singletons $\{s\}$ of the set $S$ of FSM S

Step-2: Derive the set $Q_{j+1}$ that contains each $(j+1)$-homing set of states of the FSM S that is not $j$-homing as follows:

For each subset $A$ of states of FSM S that is not in the set $\bigcup_{k=1}^{j} Q_{k}$

If there exists an input $i \in I$, such that for each $o \in O$, the $i / o$-successor of $A$ is either empty or is an item of the set $\bigcup_{k=1}^{j} Q_{k}$ then

Include the set $A$ into $Q_{j+1}$,

Derive a set $\operatorname{Tr}_{A}$ that contains each 4-tuple $\left(A, i, o, A^{\prime}\right)$ where $A^{\prime}$ is a non-empty $i / o$-successor of the set $A$;

\section{End For}

If the set of subsets $Q_{j+1}$ is empty then Return the message "there is no adaptive homing experiment for the subset $S^{\prime \prime}$.

If $Q_{j+1}$ does not contain the set $S^{\prime}$ as an item then

$j:=j+1$ and Go-to Step-2;

Step-3: Derive a homing test case $\mathrm{P}$ with the set $P$ of states as follows:

States of $P$ are subsets of states of the FSM S and the initial state of $\mathrm{P}$ is the set $S^{\prime}$; i.e., include $S^{\prime}$ into $P$;

Mark the initial state of P labeled with the set $S^{\prime}$ as a "non-visited" state in $P$;

While there is a 'non-visited' state $A$ in $P$

For each 4-tuple $\left(A, i, o, A^{\prime}\right)$ in $\operatorname{Tr}_{A}$

Add to the test case $\mathrm{P}$ as an outgoing transition of the state $A$ the transition $\left(A, i, o, A^{\prime}\right)$;

If $A^{\prime} \notin P$

Then add $A^{\prime}$ to $P$ and if $A^{\prime}$ is not a singleton, mark the added state with $A^{\prime}$ as a 'non-visited' state.

\section{EndWhile}

EndFor

If $P$ is not output-complete then

For each intermediate state $p$ of $\mathrm{P}$ where a single input $i$ is defined

If there is no transition $\left(p, i, o, p^{\prime}\right)$ for some $o \in O$ then

add a transition $\left(p, i, o, p^{\prime}\right)$ where $p^{\prime}$ is any state of $\mathrm{P}$;

\section{EndFor}

\section{Return P.}

END Procedure 1. 
Theorem 1. FSM $\mathrm{P}$ returned by Procedure 1 is a homing test case with minimal length for observable FSM $S$ if and only if the set $S^{\prime}$ is a homing set.

Proof. $\Leftarrow$ The set $S^{\prime}$ is homing if this set is $k$-homing for some $k$. For this reason, when deriving at Step $2(j+1)$-homing sets an input $i \in I$ with the desired features always exists. By construction, FSM P returned by Procedure 1 is acyclic and at each intermediate state only one input is defined with all possible outputs, i.e., $\mathrm{P}$ is a test case. At Step 3, each trace $\alpha / \beta \in \operatorname{Tr}_{\mathrm{S}}(s), s \in S^{\prime}$, that takes P to a deadlock state $\overline{s_{k}}$, takes FSM $S$ from any state of the set $S^{\prime}$ where this trace can be executed to state $s_{k}$ of $\mathrm{S}$. Thus, the test case $\mathrm{P}$ is a homing test case for FSM S.

$\Rightarrow$ Let FSM P returned by Procedure 1 be a test case for FSM S of the height $l$. By definition, in this case the set $S^{\prime}$ is a $l$-homing set, i.e. $S^{\prime}$ is a homing set.

According to Procedure 1 , if $S^{\prime}$ is an $l$-homing but it is not (l-1)-homing, then the procedure returns a test case (Step-3) of length $l$ (the set $Q_{l}$, Step-2) that is a test case of minimal length (Proposition 5).

Example. Consider the FSM S in Fig. 2. At Step-1, $Q_{0}=\{\{1\},\{2\},\{3\},\{4\}\}$. Then, at Step-2, the set $Q_{1}=\{\{1,4\},\{2,3\},\{3,4\},\{2,4\},\{2,3,4\}\}$ as by direct inspection, one can assure that the sets $\{1,4\},\{2,3\},\{3,4\},\{2,4\}$ and $\{2,3,4\}$ are

1-homing with the corresponding sets of transitions $\operatorname{Tr}_{\{1,4\}}=\{(\overline{1,4}, b, 0, \overline{1}),(\overline{1,4}$, $b, 1, \overline{2})\}, \operatorname{Tr}_{\{2,3\}}=\{(\overline{2,3}, b, 1, \overline{2}),(\overline{2,3}, b, 0, \overline{3})\}, \operatorname{Tr}_{\{3,4\}}=\{(\overline{3,4}, b, 1, \overline{2}),(\overline{3,4}$, $b, 0, \overline{3})\}, \operatorname{Tr}_{\{2,4\}}=\{(\overline{2,4}, b, 1, \overline{2})\}$ and $\operatorname{Tr}_{\{2,3,4\}}=\{(\overline{2,3,4}, b, 1, \overline{2}),(\overline{2,3,4}, b, 0$, $\overline{3})\}$. As the set $Q_{1}$ does not contain the set $S^{\prime}=\{1,2,3\}$ of the FSM S, we go back to Step-2, and then observe that the subset $\{1,2,3\}$ is 2-homing with the corresponding set of transitions $\operatorname{Tr}_{\{1,2,3\}}=\{(\overline{1,2,3}, a, 1, \overline{2,4}),(\overline{1,2,3}, a, 0, \overline{2,3})\}$. The corresponding homing test case derived by Procedure 1 for the FSM $S$ is presented in Fig. 5. Therefore, the distinguishing test case in Fig. 1 is not a shortest homing test case for the FSM S, as there exists a homing test case for S of length 2. A distinguishing test case returned by Procedure 1 for the FSM S is a test case in Fig. 1.

We now add some comments how Procedure-1 can be optimized. Step-2 of Procedure 1 can be performed by an exhaustive search of all subsets and of all possible inputs with all possible output responses. To optimize this search at each step the subsets with minimal cardinality can be checked first. The reason is that if a subset $A$ is not $j$-homing, thus each superset of $A$ is not $j$-homing too. Therefore, when checking all subsets of S states that are not in the set $\bigcup_{k=1}^{j} Q_{k}$ an optimal solution can be to start with pairs of states, then turn to triples, 4-tuples, etc.

For the same reason, at Step-2 only maximal subsets $A$ can be included into the set $Q_{j+1}$, i.e., if $A$ contains a subset $B \subset A$ that is $(j+1)$-homing then $B$ is not included into the set $Q_{j+1}$. In this case, given a 4-tuple $(A, i, o, B)$ where $B$ is an $i / o$ successor of $A$, 
the set $B$ not necessary is in the set $\bigcup_{k=1}^{j} Q_{k}$; for this reason, we add to the set $\operatorname{Tr}_{A}$ a transition $\left(A, i, o, A^{\prime}\right)$ where $A^{\prime} \in \bigcup_{k=1}^{j} Q_{k}$ and $B \subset A^{\prime}$.

The lower bound on the height of a shortest adaptive homing/distinguishing experiment significantly depends on the number of initial states of the observable FSM under experiment.

Theorem 2. Given a complete observable FSM S with $n$ states and $m$ initial states, the lower bound on the length of a shortest homing/distinguishing test case is $\sum_{i=2}^{m} C_{n}^{i}$.

Proof. If an FSM S is observable, then the corresponding lower bound on the length of adaptive experiment is proven for homing experiments in [18]. Since the length of adaptive distinguishing experiment is bounded by the same number of different subsets of states with cardinality $(m-1),(m-2), \ldots, 2$, thus, the lower bound is exactly $\sum_{i=2}^{m} C_{n}^{i}$.

Additional research is needed in order to check whether the bound $2^{n}-n-1$ is tight for distinguishing test cases and for homing test cases for machines that have non-homing pairs of states. For distinguishing test cases, the upper bound seems to be exponential. Below we show that there exists an observable FSM with four states, all of which are initial states, such that the longest trace in the distinguishing test case traverses all subsets with at least two states, i.e., its length equals $11=2^{4}-4-1$.

Consider an FSM S with the flow table in Table 1 and with the set $\{1,2,3,4\}$ of initial states. The FSM S has 11 inputs and four outputs. The inputs are defined $x_{123}$, $x_{124}, x_{134}, \ldots, x_{12}$ to demonstrate which subset is traversed when applying this input. An input $x_{123}$ defines $x_{123} / 0$-successor of the subset $\{1,2,3,4\}$ in the distinguishing test case, this successor coincides with the index of the input, i.e. it is the set $\{1,2,3\}$.

Table 1. Flow Table of the FSM S

\begin{tabular}{|c|c|c|c|c|}
\hline$x \backslash s$ & 1 & 2 & 3 & 4 \\
\hline$x_{123}$ & $1 / 0,2,3$ & $2 / 0,2,4$ & $3 / 0,3,4$ & $4 / 2,3,4$ \\
\hline$x_{124}$ & $1 / 0,1$ & $2 / 0,1$ & $4 / 0$ & $3 / 0 ; \quad 4 / 1$ \\
\hline$x_{134}$ & $1 / 0,1$ & $3 / 0$ & $2 / 0 ; \quad 3 / 1$ & $4 / 0,1$ \\
\hline$x_{234}$ & $2 / 0 ; \quad 1 / 2$ & $1 / 0 ; 2 / 1$ & $3 / 0,2$ & $4 / 0,1$ \\
\hline$x_{34}$ & $1 / 1,2,4 ; 2 / 5$ & $3 / 2 ; \quad 2 / 4,5$ & $3 / 0,1,4$ & $4 / 0,1,5 ; \quad 2 / 2$ \\
\hline$x_{24}$ & $1 / 0,1,2$ & $3 / 0 ; \quad 2 / 1$ & $2 / 0 ; \quad 3 / 2$ & $4 / 0 ; 3 / 1$ \\
\hline$x_{14}$ & $2 / 0 ; \quad 1 / 1$ & $1 / 0$ & $3 / 0,1$ & $4 / 0,1$ \\
\hline$x_{13}$ & $1 / 0,1$ & $2 / 0,1,2$ & $4 / 1 ; 3 / 0$ & $3 / 0,1 ; \quad 4 / 2$ \\
\hline$x_{23}$ & $2 / 0,3 ; \quad 1 / 1$ & $1 / 0 ; \quad 2 / 1,2$ & $3 / 0,23$ & $3 / 1 ; \quad 4 / 2,3 ; \quad 1 / 0$ \\
\hline$x_{12}$ & $3 / 0 ; \quad 1 / 1$ & $2 / 0,1$ & $1 / 0 ; \quad 3 / 2$ & $4 / 0,1,2$ \\
\hline$x_{\text {sep }}$ & $1 / 0,2$ & $2 / 1,3$ & $2 / 1 ; \quad 1 / 2$ & $1 / 0 ; \quad 2 / 1$ \\
\hline
\end{tabular}


The two last state subsets that are traversed by a longest trace in the distinguishing test case are $\{2,3\}$ and $\{1,2\}$. The set $\{1,2\}$ is an $x_{12} / 0$-successor of the subset $\{2,3\}$ in the test case. The last input $x_{\text {sep }}$ separates states 1 and 2 of the FSM S and takes the test case to different 0-distinguishing sets. We further illustrate how this distinguishing test case can be derived for the FSM S using a Procedure similar to Procedure 1.

We first derive all 0-distinguishing sets that are singletons $\{1\},\{2\},\{3\},\{4\}$. according to the Procedure similar to Procedure 1, we then derive a set $Q_{1}$, and one can assure that there is a single 1-distinguishing set for the FSM S. This is the set $\{1,2\}$ that is included into the set $Q_{1}$. The set $Q_{2}$ contains a single set $\{2,3\}$, the set $\{1,3\}$ is a single 3 -distinguishing set that is included into the set $Q_{3}$, etc. Thus, one can iteratively derive all $Q_{1}, Q_{2}, Q_{3}, \ldots$, sets, till reaching the set $Q_{11}$ that contains the set $\{1,2,3,4\}$ of initial states. A shortest distinguishing experiment traverses all nonempty subsets of the set $\{1,2,3,4\}$ and thus, has the length 11 . The longest trace of the experiment covers the chain of sets $\{1,2,3,4\},\{1,2,3\},\{1,2,4\},\{1,3,4\},\{2$, $3,4\},\{3,4\},\{2,4\},\{1,4\},\{1,3\},\{2,3\}$, and $\{1,2\}$, respectively. All other traces do not allow to uniquely determine a state of the FSM before the experiment and thus, since a sequence of all subsets of some set is traversed by a trace of a test case of minimal length it seems that the exponential upper bound can be reached for adaptive experiments with observable nondeterministic FSMs.

\section{Conclusion}

Given a non-initialized complete nondeterministic observable FSM, a method for deriving adaptive homing/distinguishing experiments is proposed. Adaptive experiments are represented as special nondeterministic observable machines, called test cases, and necessary and sufficient conditions for having adaptive homing/distinguishing test cases with minimal length for observable nondeterministic FSMs are presented. The lower bound on the length of shortest homing/distinguishing test cases is evaluated. Possible extensions to the proposed work include extending the proposed method for non-observable FSMs and adapting the work to partial nondeterministic FSMs by extending related work in [23] and to timed nondeterministic FSMs based on the work presented in [28]. Also it would be interesting to determine the tight lower bound on the length of shortest homing/distinguishing test cases.

\section{References}

1. Bochmann, G.V., Petrenko, A.: Protocol testing: review of methods and relevance for software testing. In: Proc. of International Symposium on Software Testing and Analysis, Seattle, pp. 109-123 (1994)

2. Dorofeeva, R., El-Fakih, K., Maag, S., Cavalli, A.R., Yevtushenko, N.: FSM-based conformance testing methods: a survey annotated with experimental evaluation. Information and Software Technology 52, 1286-1297 (2010)

3. Gill, A.: State-identification experiments in finite automata. Information and Control, 132$154(1961)$ 
4. Kohavi, Z.: Switching and Finite Automata Theory. McGraw-Hill, New York (1978)

5. Lee, D., Yannakakis, M.: Testing finite-state machines: state identification and verification. IEEE Trans. on Computers 43(3), 306-320 (1994)

6. Lee, D., Yannakakis, M.: Principles and methods of testing finite state machines-a survey. Proceedings of the IEEE 84(8), 1090-1123 (1996)

7. Simao, A., Petrenko, A., Maldonado, J.C.: Comparing finite state machine test. IET Software 3(2), 91-105 (2009)

8. Moore, E.F.: Gedanken-experiments on sequential machines. In: Automata Studies (Annals of Mathematical Studies no.1), pp. 129-153. Princeton University Press (1956)

9. Hennie, F.C.: Fault detecting experiments for sequential circuits. In: Proc. of 5th Annual Symposium on Switching Circuit Theory and Logical Design, pp. 95-110. Princeton (1964)

10. Petrenko, A., Simao, A., Yevtushenko, N.: Generating Checking Sequences for Nondeterministic Finite State Machines. In: Proc. of ICST 2012, pp. 310-319 (2012)

11. Mathur, A.: Foundations of Software Testing. Addison Wesley (2008)

12. Agibalov, G., Oranov, A.: Lectures on Automata Theory. Tomsk State University Publishers (1984) (in Russian)

13. Ginsburg, S.: On the length of the smallest uniform experiment which distinguishes the terminal states of a machine. Journal of the ACM 5(3), 266-280 (1958)

14. Hibbard, T.N.: Lest upper bounds on minimal terminal state experiments of two classes of sequential machines. Journal of the ACM 8(4), 601-612 (1961)

15. Sandberg, S.: Homing and Synchronization Sequences. In: Broy, M., Jonsson, B., Katoen, J.-P., Leucker, M., Pretschner, A. (eds.) Model-Based Testing of Reactive Systems. LNCS, vol. 3472, pp. 5-33. Springer, Heidelberg (2005)

16. Ravikumar, B.: Parallel algorithms for finite automata problems. In: Rolim, J.D.P. (ed.) IPPS-WS 1998 and SPDP-WS 1998. LNCS, vol. 1388, p. 373. Springer, Heidelberg (1998)

17. Spitsyna, N., El-Fakih, K., Yevtushenko, N.: Studying the separability relation between finite state machines. Software Testing, Verification and Reliability 17(4), 227-241 (2007)

18. Kushik, N., El-Fakih, K., Yevtushenko, N.: Preset and Adaptive Homing Experiments for Nondeterministic Finite State Machines. In: Bouchou-Markhoff, B., Caron, P., Champarnaud, J.-M., Maurel, D. (eds.) CIAA 2011. LNCS, vol. 6807, pp. 215-224. Springer, Heidelberg (2011)

19. Starke, P.: Abstract Automata. American Elsevier (1972)

20. Kushik, N., Yevtushenko, N.: On the Length of Homing Sequences for Nondeterministic Finite State Machines. In: Konstantinidis, S. (ed.) CIAA 2013. LNCS, vol. 7982, pp. 220 231. Springer, Heidelberg (2013)

21. Zhang, F., Cheung, T.: Optimal Transfer Trees and Distinguishing Trees for Testing Observable Nondeterministic Finite-State Machines. IEEE Transactions on Software Engineering 19(1), 1-14 (2003)

22. Alur, R., Courcoubetis, C., Yannakakis, M.: Distinguishing tests for nondeterministic and probabilistic machines. In: Proc. of the 27th ACM Symposium on Theory of Computing, pp. 363-372 (1995)

23. Petrenko, A., Yevtushenko, N.: Conformance Tests as Checking Experiments for Partial Nondeterministic FSM. In: Grieskamp, W., Weise, C. (eds.) FATES 2005. LNCS, vol. 3997, pp. 118-133. Springer, Heidelberg (2006)

24. Gromov, M.L., Evtushenko, N.V., Kolomeets, A.V.: On the Synthesis of Adaptive Tests for Nondeterministic Finite State Machines. Progr. and Comp. Software 34(6), 322-329 (2008) 
25. Petrenko, A., Yevtushenko, N.: Adaptive Testing of Deterministic Implementations Specified by Nondeterministic FSMs. In: Wolff, B., Zaïdi, F. (eds.) ICTSS 2011. LNCS, vol. 7019, pp. 162-178. Springer, Heidelberg (2011)

26. Tretmans, J.: Model-Based Testing with Labelled Transition Systems: There is nothing More Practical than a Good Theory. Slides from the lecture at TAROT Summer School (2010), http: / / tarot2010.ist.tugraz.at/

27. Gromov, M., El-Fakih, K., Shabaldina, N., Yevtushenko, N.: Distinguing nondeterministic timed finite state machines. In: Lee, D., Lopes, A., Poetzsch-Heffter, A. (eds.) FMOODS/FORTE 2009. LNCS, vol. 5522, pp. 137-151. Springer, Heidelberg (2009)

28. El-Fakih, K., Gromov, M., Shabaldina, N., Yevtushenko, N.: Distinguishing experiments for timed nondeterministic finite state machines. Acta Cybernetica (to appear)

29. Petrenko, A., Yevtushenko, N., Bochmann, G.V.: Testing Deterministic Implementations from their Nondeterministic Specifications. In: Proc. of the IFIP Ninth International Workshop on Testing of Communicating Systems, pp. 125-140 (1996) 